\title{
Conformational Study of Polypeptide Chains Grafted on the Surface of Polylactide Latex Particle
}

\author{
Satoshi Tanimoto, ${ }^{1}$ Toshiya Iwata, ${ }^{1}$ Hitoshi Yamaoka, ${ }^{1}$ Masahiro Yamada, ${ }^{2}$ and Kana Kobori ${ }^{2}$ \\ ${ }^{1}$ Department of Materials Science, The University of Shiga Prefecture, 2500 Hassaka, Hikone 522-8533, Japan \\ ${ }^{2}$ Advanced Materials Development Center, Osaka Gas Chemicals Co., Ltd., 5-11-61 Torishima, Konohana-ku, Osaka 554-0051, Japan
}

Correspondence should be addressed to Satoshi Tanimoto, tanimoto@mat.usp.ac.jp

Received 22 July 2009; Accepted 3 September 2009

Recommended by Vladimir Tsukruk

Polylactide (PLA) latex particle covered with polypeptide chains were prepared by means of solvent exchange method from PLA and PLA-block-polypeptide block copolymer solutions. PLA segment of the block copolymer and PLA homopolymer formed a core of the particle, and the polypeptide segment of the block copolymer, which is designed as tightly fixed biodegradable emulsifier, formed corona around the particle surface. This picture was supported by the fact that zeta-potential of PLA latex particle covered with polypeptide segment was different from that of bare PLA particle because of the presence of the ionizable group in the polypeptide chains. To clarify the effect of the ionizable group on conformation of the polypeptide chain, the relation between the polypeptide chain length and the area occupied by the single block chain was evaluated. The result that the occupied area per a polypeptide chain was linearly increased with the increase in the polypeptide chain length indicates that the polypeptide chains trail on the particle surface and did not take helical structures.

Copyright ( $) 2009$ Satoshi Tanimoto et al. This is an open access article distributed under the Creative Commons Attribution License, which permits unrestricted use, distribution, and reproduction in any medium, provided the original work is properly cited.

\section{Introduction}

To control global warming, it is important to convert not only a source of energy but also raw materials in various industries to the biomass from the petroleum. Polylactide and polypeptide are the biomass-originated materials, which are obtained from polymerization of lactic acid or amino acid. In addition, they are known as biocompatible and biodegradable materials. Much research aimed at applications of PLA materials and peptide derivatives has been carried out. For example, a PLA scaffold for bone regeneration and a PLA screw for bone fixation as an application of the biodegradable characteristics has been already examined [1-3]. Many groups have applied the biocompatibility of polypeptides to a wound dressing [4], MRI contrast agents [5], and tissue engineering [6]. However, an application range of PLA materials has been quite limited in the fields such as noted above. Therefore, we have paid attention to PLA latex dispersions since a brand-new style of PLA materials should be necessary to expand the applicable field. Assuming that a perfectly biodegradable or biocompatible latex dispersion is available, it becomes possible for us to develop new biomaterials such as biocompatible glue and a biocompatible paint.

In this study, amphiphilic block copolymers, PLA-blockpolypeptide, were synthesized from PLA and hydrophilic polypeptide, and their application to a biodegradable surfactant was examined. The surfactant was used to stabilize a PLA latex dispersion since it had biodegradable and biocompatible characteristics. The PLA-block-polypeptide and the PLA homopolymer were self-organized with a solvent exchange method [7] in order to anchor the hydrophilic polypeptide chains which were the source of surface stabilization. Moreover, we discussed the conformation of polypeptide chain which was grafted on the surface of latex particle and which have determined the stability of the latex dispersion.

\section{Experimental}

2.1. Materials. Amino acid used in this study, $\mathcal{E}$-(benzyloxycarbonyl)-L-lysine (L-lys $(\mathrm{Z})$ ), was purchased from 
BACHEM and used without further purification. Triphosgene was purchased from Nacalai Tesque, Inc. 33wt $\%$ hydrobromide/acetic acid solution was purchased from Aldrich and used as received. Polylactide (PLA, MW: 5000), N,N'-dicyclohexylcarbodiimide (DCC), and N-hydroxysuccinimide (NHS) were purchased from Wako Pure Chemical Industries, Ltd.

2.2. Synthesis of PLA-block-poly(L-lysine). L-Lys(Z) N-carboxyanhydride (L-lys(Z)-NCA) was obtained from L-lys(Z) by the Fuchs-Farthing method using triphosgene [8]. Poly(Llys $(Z)$ ) was synthesized in DMF by a ring-opening polymerization of L-lys(Z)-NCA using n-propylamine as an initiator [9]. The polypeptide was then coupled with PLA homopolymer with DCC and NHS in DMF [10]. The mole ratio of them was $1: 1: 5: 5$, respectively. Zgroup used as an amino-protecting group of polypeptide side chain was cleaved by 33\% hydrobromide/acetic acid solution. The concentration of the cleaving reagent was determined according to [10]. All the steps were carried out under $\mathrm{N}_{2}$ atmosphere. The final product was purified by reprecipitation in over 50 -fold diethylether, filtered, and dried in vacuum. The copolymer obtained through these synthesis procedures was named as a peptide copolymer in this paper.

2.3. Particle Preparation. PLA latex particle was prepared from PLA homopolymer and PLA-block-poly(L-lysine) block copolymer by the solvent exchange method as shown in Figure 1 [7]. At first, PLA was dissolved in THF at a concentration of $1 \mathrm{mg} / \mathrm{mL}$. Then, aqueous solution of the peptide copolymer was slowly added to the PLA solution in THF with a constant feed rate and the final ratio of the peptide copolymer and PLA was adjusted from $0.5 \%$ to $20 \%$. The mixed solution was kept at $30^{\circ} \mathrm{C}$ for overnight under dust-free atmosphere to evaporate THF portion. After completion of evaporation of THF, PLA particulate coated with polypeptide was obtained in the form of latex dispersion in water.

2.4. Characterization. Characterizations of the polymers were obtained by a gel permeation chromatograph (GPC) (Series-10A, Shimadzu Corporation) with polystyrene columns (Polymer Laboratory, Mixed-C and Mixed-E) and by a nuclear magnetic resonance (NMR) (Lambda-400, Jeol Ltd.). Particle diameter of PLA/peptide copolymer latex was measured by a dynamic light-scattering (DLS) apparatus (DLS-7000, Otsuka Electronics, Japan) with Marquart calculation. Zeta-potential of the latex particle was evaluated in water with an electrophoretic light scattering (ELS) apparatus (ELS-Z2, Otsuka Electronics, Japan). Morphological observation of latex particles was performed with a scanning electron microscope (SEM) (VE-9800, Keyence Corporation, Japan).

\section{Results and Discussion}

3.1. Sample Characterization. Copolymers employed in this study were characterized by GPC and NMR. The molecular

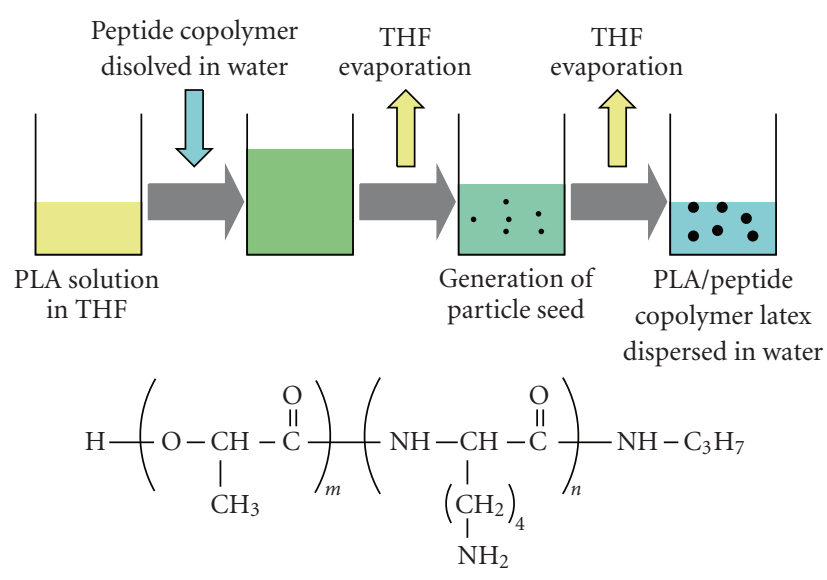

Chemical structure of peptide copolymer

Figure 1: Particle preparation procedure and chemical structure of peptide copolymer used in this study. The peptide-coated PLA latex dispersion was obtained by the solvent exchange technique.

TABle 1: Molecular characteristics of copolymer and PLA homopolymer used in this study.

\begin{tabular}{lccc}
\hline Sample code & PLA:P $(\text { Lys })^{1)}$ & M.W. $^{1)}$ & Mw/Mn \\
\hline L-K9 & $70: 09$ & 6086 & 1.30 \\
L-K20 & $70: 20$ & 7341 & 1.30 \\
L-K31 & $70: 31$ & 9132 & 1.24 \\
L-K46 & $70: 46$ & 11054 & 1.15 \\
L-K76 & $70: 76$ & 13735 & 1.19 \\
L-K123 & $70: 123$ & 19101 & 1.20 \\
PLA & N/A & 5000 & 1.20 \\
\hline
\end{tabular}

1) calculated from ${ }^{1} \mathrm{H}$ NMR spectra, 2) measured by GPC.

weight and the polydispersity index of the copolymers were summarized in Table 1. The number of the sample code in Table 1 shows the polymerization degree of the polypeptide block chain part in the copolymer. The polymerization degree of PLA segment in the peptide copolymer was fixed at 70, in order to investigate the chain length effect of the polypeptide on the surface property of the particle. SEM microphotograph of the particles prepared with the peptide copolymer (not shown here) indicated that the shape of the particles was spherical.

Figure 2 showed the size distribution of the particle made with the peptide copolymer L-K31 measured by DLS. The average diameter and the standard deviation were calculated as $303.5 \mathrm{~nm}$ and $72.8 \mathrm{~nm}$, respectively. The particles prepared with other peptide copolymer with different polypeptide length showed similar size distribution.

Stabilization of the dispersion was estimated by turbidity measured at $550 \mathrm{~nm}$ of wavelength. (Results are not shown.) The turbidity of the PLA latex dispersion prepared without the peptide copolymer decreased rapidly, and after 3 days the dispersion became transparent. On the other hand, the dispersion made with the peptide copolymer was kept turbid for over 10 days. From this result, it was revealed that the existence of peptide copolymer improved dispersion stability. 


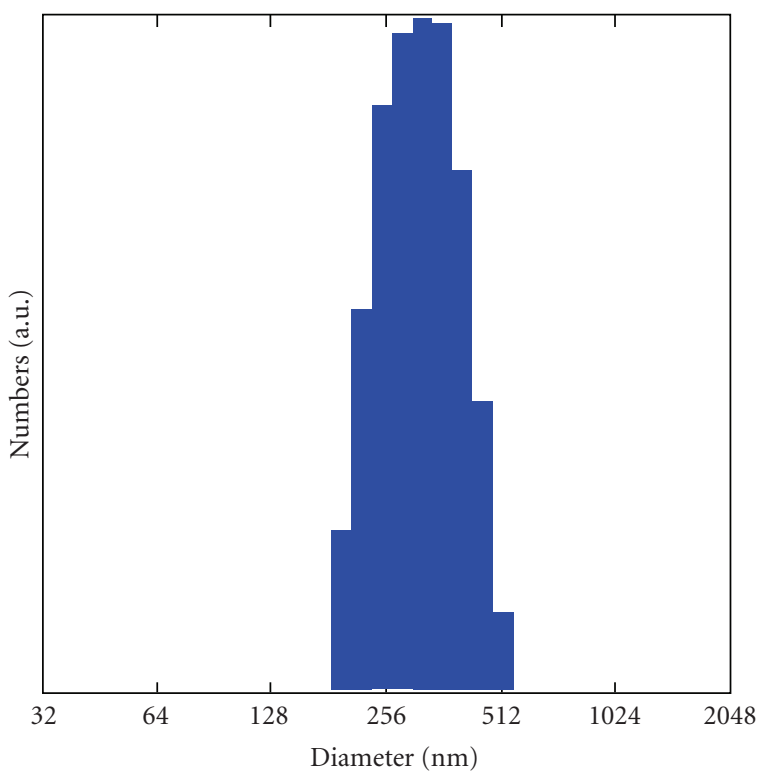

Figure 2: An example of size distribution profile obtained from DLS measurement for the PLA latex particle made with the copolymer L-K31.

3.2. Surface Peptide Density and Zeta-Potential. To estimate the effect of the peptide copolymer on the electrostatic surface property of PLA particle, zeta-potential was measured for the particles with various copolymer fractions. As the fraction of the peptide copolymer in making PLA latex increased, zeta-potential of the PLA particle increased and became constant over 0.10 of the peptide copolymer fraction. The similar tendency was observed for all the other copolymers having a variety of the polypeptide chain length. We assumed that the stagnation of zeta-potential was due to saturation of peptide copolymers on the particle surface. In order to prove this assumption, we estimated surface density of polypeptide on the PLA particle. The surface polypeptide density was calculated with

$$
D=\frac{N}{A}=\frac{N_{0}}{A N_{p}},
$$

where $D$ is the surface density of polypeptide as a quotient of $N$ and $A$ which is a surface area of one particle, which can be calculated on the basis of the particle diameter obtained from DLS measurement. Also $N$ is a number of copolymer chains per one particle and is able to be mathematically calculated as a ratio of a total number of copolymer chains in the system, $N_{0}$ to a total number of the particle, $N_{P}$ which can be obtained as a ratio of a total PLA volume in the system to a volume for one particle calculated from its diameter. The final quotient, $D$ is a surface density of the peptide copolymer when the peptide copolymer is assumed to be concentrated only on the surface region of the particle.

Figure 3 shows a relation between the surface density of the polypeptide chain and zeta-potential of the particle. In this study, the most effective factor to determine the zetapotential of the particle is considered to be a number of

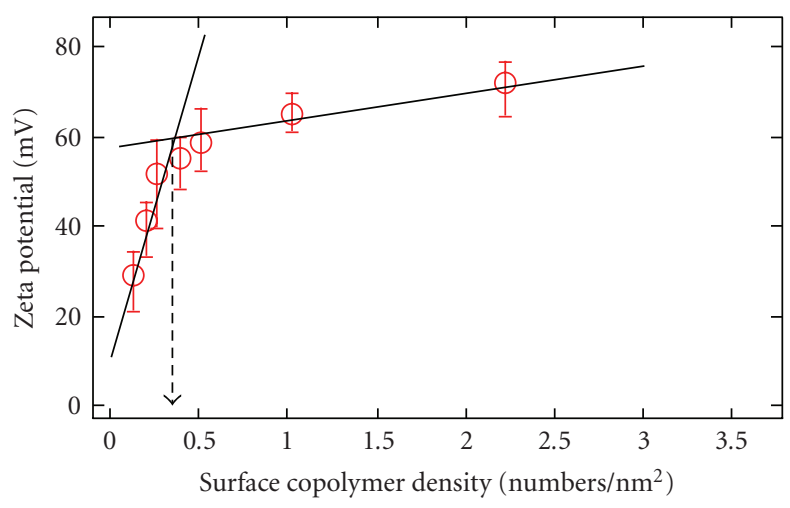

FIgUre 3: Zeta-potential of the PLA particles stabilized by peptide copolymer L-K9 as a function of the surface density of peptide chain.

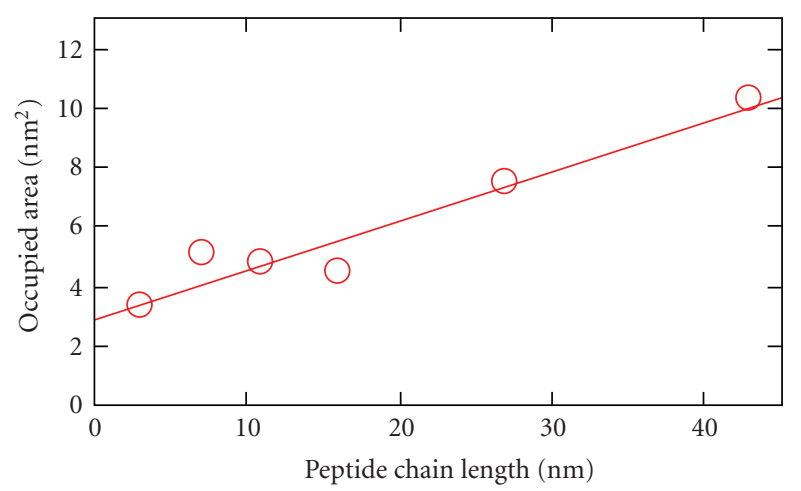

FIGURE 4: Occupation area of one polypeptide chain as a function of polypeptide length calculated from their polymerization degree.

amino groups at the end of lysine side chain. Therefore, the plateau observed at the high surface copolymer density region in Figure 3 indicates that the polypeptide chains covered a whole surface of the PLA particle. Then, the two tangential lines shown in Figure 3 made the intersection, which gave the surface density of the polypeptide chain in saturated condition. The reciprocal of the saturated surface density was defined as the area occupied by one polypeptide chain.

Figure 4 shows a plot of the area occupied by one polypeptide chain as a function of the polypeptide length. Notice that the occupation area increases linearly with increase of the polypeptide chain length. If the polypeptide chain takes a helical structure standing on the PLA particle surface, the polypeptide segment probably occupies constant area $\left(2.3 \mathrm{~nm}^{2}\right)$ irrespective of the polypeptide length. The results shown in Figure 4, however, show the dependence of the area on the polypeptide length, which suggests that the polypeptide conformation was not standing perpendicular to the surface with a helical structure but was lying on the particle surface. In order to confirm the polypeptide conformation, a circular dichroism (CD) measurement was carried out, and the results for the PLA latex particles 


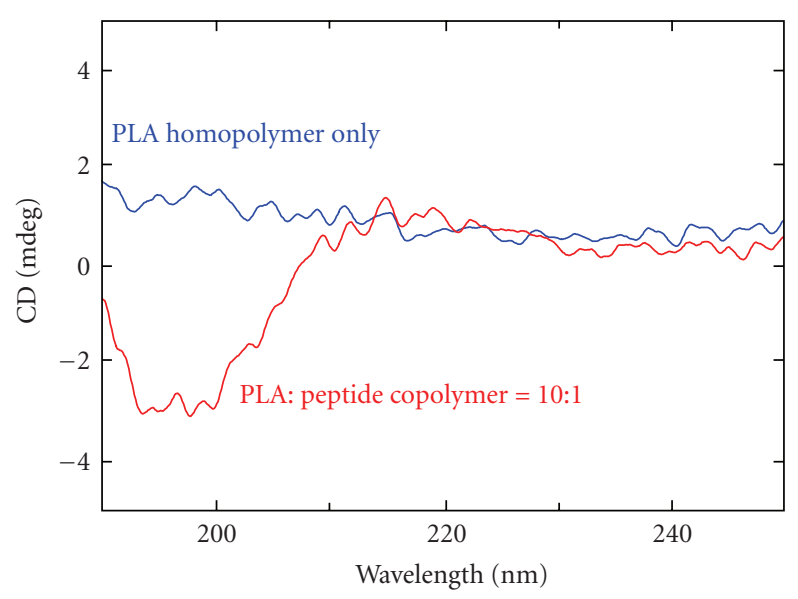

FIGURE 5: CD spectra of the particles made with and without the peptide copolymer L-K46.

prepared with/without the peptide copolymer were shown in Figure 5. The CD spectrum plotted in red was the result of the particle prepared with the peptide copolymer, and showed a negative and a positive cotton effect at $198 \mathrm{~nm}$ and at $218 \mathrm{~nm}$, respectively. This result also supports the assumption that the peptide chain was in a random conformation [11, 12]. And if the peptide chain takes a random coil structure and spreads out three-dimensionally, the occupied area must increase in proportional to the power three halves of peptide chain length. This assumption does not match with a linear increase shown in Figure 4. It is presumably understood by taking it into account that the polypeptide chain expands 2-dimensionally in the vicinity of the particle surface. This model might be more acceptable in consideration of the electrostatic interaction between ionized carboxyl groups of PLA surfaces and protonized amino groups of lysine side branches.

\section{Conclusion}

We synthesized amphiphilic polylactide-block-poly(L-lysine) copolymers and succeeded in a preparation of PLA particles coated with hydrophilic peptide chains via solvent exchange method. The polypeptide chains on the surface of PLA particle were revealed to be a random structure and to be covering a particle surface. The conformational information on the polypeptide chains obtained in this study will provide fundamental guidelines on designing PLA particles having a highly functionalized surface.

\section{Acknowledgment}

A useful discussion with Professor Yoshitsugu Hirokawa of The University of Shiga Prefecture, Japan, concerning conformational analysis of the peptide is gratefully acknowledged.

\section{References}

[1] M. Kellomäki, H. Niiranen, K. Puumanen, N. Ashammakhi, T. Waris, and P. Törmälä, "Bioabsorbable scaffolds for guided bone regeneration and generation," Biomaterials, vol. 21, no. 24, pp. 2495-2505, 2000.

[2] K. C. Yerit, S. Hainich, D. Turhani, et al., "Stability of biodegradable implants in treatment of mandibular fractures," Plastic and Reconstructive Surgery, vol. 115, no. 7, pp. 18631870, 2005.

[3] H. W. Losken, J. A. van Aalst, M. P. Mooney, et al., "Biodegradation of Inion fast-absorbing biodegradable plates and screws," Journal of Craniofacial Surgery, vol. 19, no. 3, pp. 748-756, 2008.

[4] R. Eloy, A. Brack, N. Dorme, and A. M. Cornillac, "Physical and biological properties of a new synthetic amino acid copolymer used as wound dressing," Journal of Biomedical Materials Research, vol. 26, no. 6, pp. 695-712, 1992.

[5] N. Nitin, L. E. W. LaConte, O. Zurkiya, X. Hu, and G. Bao, "Functionalization and peptide-based delivery of magnetic nanoparticles as an intracellular MRI contrast agent," Journal of Biological Inorganic Chemistry, vol. 9, no. 6, pp. 706-712, 2004.

[6] C. E. MacPhee and D. N. Woolfson, "Engineered and designed peptide-based fibrous biomaterials," Current Opinion in Solid State and Materials Science, vol. 8, no. 2, pp. 141-149, 2004.

[7] H. Yabu, T. Higuchi, K. Ijiro, and M. Shimomura, "Spontaneous formation of polymer nanoparticles by good-solvent evaporation as a nonequilibrium process," Chaos, vol. 15, no. 4, Article ID 047505, 7 pages, 2005.

[8] A. Harada and K. Kataoka, "Formation of polyion complex micelles in an aqueous milieu from a pair of oppositelycharged block copolymers with poly(ethylene glycol) segments," Macromolecules, vol. 28, no. 15, pp. 5294-5299, 1995.

[9] T. J. Deming, "Living polymerization of $\alpha$-amino acid-Ncarboxyanhydrides," Journal of Polymer Science Part A, vol. 38, no. 17, pp. 3011-3018, 2000.

[10] E. B. Lavik, J. S. Hrkach, N. Lotan, R. Nazarov, and R. Langer, "A simple synthetic route to the formation of a block copolymer of poly(lactic-co-glycolic acid) and polylysine for the fabrication of functionalized, degradable structures for biomedical applications," Journal of Biomedical Materials Research, vol. 58, no. 3, pp. 291-294, 2001.

[11] Y. P. Myer, "The pH-induced helix-coil transition of polyL-lysine and poly-L-glutamic acid and the 238-m $\mu$ dichroic band," Macromolecules, vol. 2, no. 6, pp. 624-628, 1969.

[12] H. Yamamoto and T. Hayakawa, "Synthesis and conformational study of poly(N-benzyl-L-lysine) and its benzyloxycarbonyl derivative," Biopolymers, vol. 11, no. 6, pp. 1259-1268, 1972. 

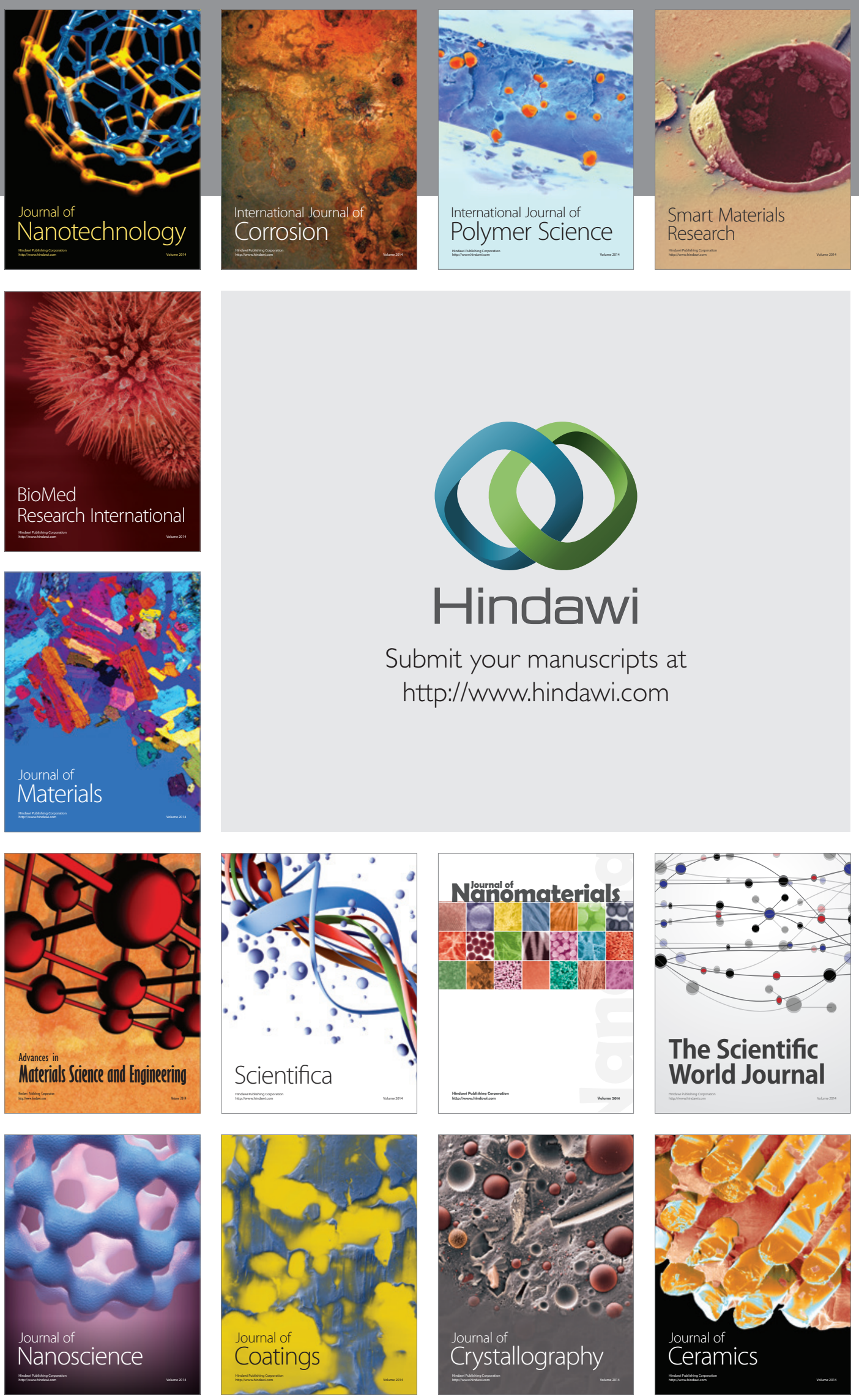

The Scientific World Journal

Submit your manuscripts at

http://www.hindawi.com

\section{World Journal}

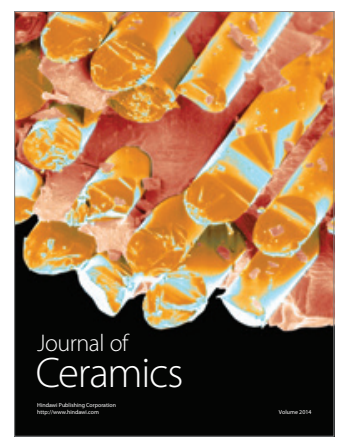

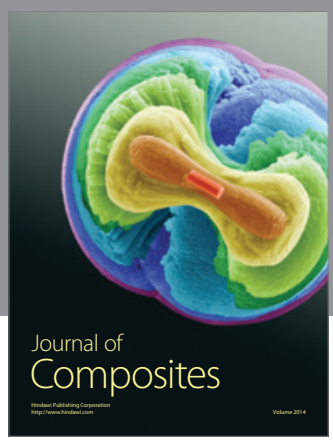
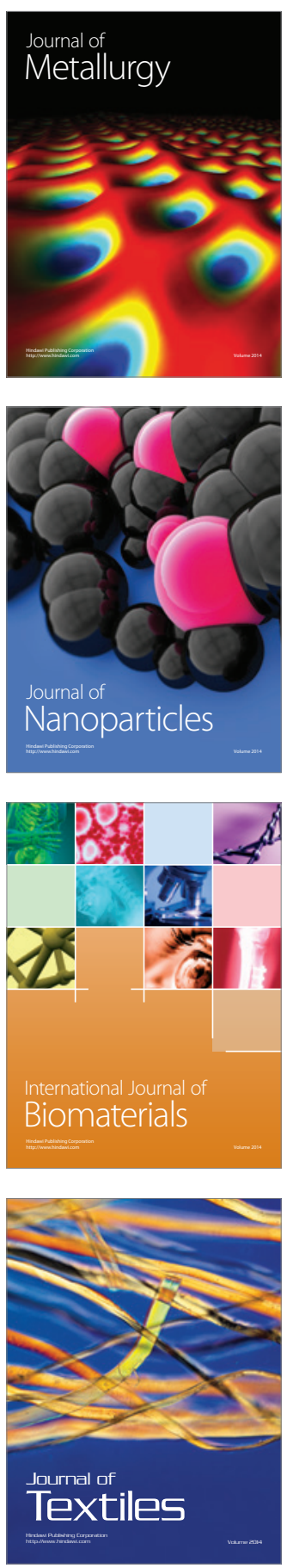